\title{
Efecto de las barreras de innovación en la capacidad de absorción de las empresas innovadoras
}

\author{
Carpio Freire, Carlos Raúl \\ Centrum Católica Graduate Business School - Pontificia Universidad Católica del Perú \\ Correo electrónico: rcarpiof@pucp.pe \\ AfCha Chávez, SERgIO MOISÉS \\ Centrum Católica Graduate Business School - Pontificia Universidad Católica del Perú \\ Correo electrónico: safcha@pucp.pe
}

\begin{abstract}
RESUMEN
El presente trabajo estudia la importancia de la capacidad de absorción de las empresas innovadoras y el efecto que en ella ejercen las barreras de innovación. Se revisa además la gestión de inversión en capacidad de absorción como promotor de innovación y se exponen las implicaciones al nivel de desempeño de las empresas en cuestiones de procesos y posición en el mercado. Se realiza el análisis mediante modelos de regresión lineal simple y múltiple. Finalmente, el impacto de la innovación en las empresas se obtuvo mediante un análisis de componentes principales.
\end{abstract}

Palabras clave: capacidad de absorción, barreras a la innovación, modelos de regresión, empresas innovadoras.

Clasificación JEL: O32.

MSC2010: 62J05; 62H25. 


\title{
Effect of innovation barriers on the absorptive capacity of innovative companies
}

\begin{abstract}
This paper studies the importance of the absorption capacity of innovative companies and the effect of innovation barriers on it. The management of absorption capacity investment as an innovation promoter is also reviewed and the implications are exposed to the level of performance of the companies in matters of processes and market position. The analysis is performed using simple and multiple linear regression models. Finally, the impact of innovation on companies was obtained through a Principal Component Analysis.
\end{abstract}

Keywords: absorption capacity, innovation barriers, regression models, innovative companies.

JEL classification: $O 32$.

MSC2010: 62J05; 62H25.

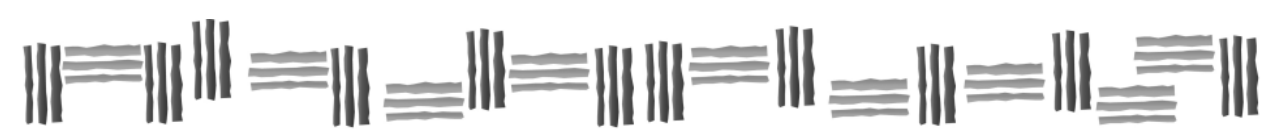




\section{Introducción.}

La necesidad de reducir la pobreza y de poseer una mayor participación en el mercado latinoamericano y mundial, ha motivado a los gobiernos y a las empresas a concentrarse en la inversión para el desarrollo del capital humano en áreas como: conocimiento, ciencia, tecnología e innovación (Govindarajan \& Trimble, 2013). Un caso reciente es el plan del gobierno de Ecuador para generar un cambio en la matriz productiva del país, con el objetivo de pasar de ser una economía primaria exportadora a ser una economía basada en el conocimiento, ha motivado una amplia inversión con fondos públicos que se destina a la creación de universidades, institutos y centros de investigación, con el propósito de obtener resultados en el desarrollo del talento humano, propuesto como el pilar fundamental del cambio de la matriz de producción de Ecuador (SENPLADES, 2012).

No son pocos los problemas encontrados para implementar estos cambios (Wagner, 2013); las empresas hacen esfuerzos continuos para enfrentar las barreras sistémicas y no sistémicas, también llamadas internas y externas que se les presentan para lograr la generación, adopción o implementación de la innovación en sus productos, procesos y su gestión (Madrid-Guijarro, García \& Van Auken, 2009). El presente trabajo propone estudiar los factores que perciben los empresarios como barreras $\mathrm{u}$ obstáculos a los procesos innovativos de las empresas, desde la gerencia estratégica de la empresa al momento de tomar decisiones observando las competencias organizacionales y la capacidad de absorción del conocimiento.

\section{Antecedentes.}

Realizar una innovación exitosa depende de la combinación adecuada de varios factores que van desde el financiero hasta las demandas del mercado, pasando por el personal capacitado, así como la adecuada relación con el entorno empresarial (Ahmed \& Shepherd, 2012). Pese a que el entorno político y jurídico puede presentar normas y proyectos de incentivo a la mejora y diversificación de procesos y productos, e incluso estimular el desarrollo de un adecuado sistema nacional de innovación, algunas empresas se abstienen de iniciar procesos innovativos. Esto, debido al reto y esfuerzo que dicho proceso implica; pero además, por encontrar factores que pueden percibirse como barreras que bloquean o inhiben dichos procesos (Woolthuis, Lankhuizen, \& Gilsing, 2005).

El presente trabajo plantea examinar dichos factores, los tipos que se presentan y su relación con los procesos innovativos llevados a cabo por las compañías, aplicando el proceso de estudio científico acorde a la normativa de investigación en las ciencias administrativas (Sekaran \& Bougie, 2009). Trabajos relevantes en esta área son los de Damanpour, Szabat y Evan (1989) estudiando la relación entre los diferentes tipos de innovación y el rendimiento organizacional; Cohen y Levinthal (1990) abordando el estudio de las capacidades absortivas; Arundel (1997) sobre las estrategias de las empresas frente a los factores que obstaculizan la innovación; D'Este, Iammarino y von Tunzelmann (2012), analizando la percepción de estos factores como barreras y su influencia sobre las prioridades en políticas de fomento a la innovación (Holzl \& Janger, 2013), aportando a la escasa literatura que sobre el tema hay en Latinoamérica, posibilitando nuevos estudios comparativos con otros conglomerados empresariales de la región.

\section{Marco Teórico.}

\subsection{Competencias organizacionales.}

Una de las preguntas más importantes en la administración estratégica es cómo las empresas logran ventajas competitivas de manera sostenible (Barney, Della, Sciarelli \& Arikan, 2012). La generación de valor y ventajas competitivas son producto de las capacidades de aprendizaje que poseen las firmas 
para procesar información obtenida de fuentes externas, entender nuevas ideas e implementarlas con el fin de mejorar los resultados de la empresa (Cohen \& Levinthal, 1990).

Wang, Hermens, Huang y Chelliah (2015) demuestran la influencia positiva y significativa del conocimiento organizacional y la capacidad de aprendizaje y en el desempeño de las empresas. En virtud de ello, estudios teóricos han analizado cómo el conocimiento organizacional es adquirido, retenido, transformado y transmitido dentro de la empresa (Petersen, Pedersen \& Sharma, 2003). Casillas, Moreno, Acebo, Gallego y Ramos (2009) proponen un modelo del proceso de gestión del conocimiento en las empresas que incluye: (a) conocimiento previo, (b) adquisición de nuevo conocimiento, (c) integración de conocimiento nuevo con el conocimiento previo, (d) acción y (e) retroalimentación.

Cuando se habla de aprendizaje organizacional corresponde a un proceso basado en el aprendizaje individual, a través del cual la organización genera conocimiento con el fin de adaptarse a las condiciones cambiantes del entorno (Castañeda, 2015). Para Chiva y Alegre (2005) el aprendizaje organizacional puede ser entendido como el proceso de construcción social dentro de la empresa en donde se comparten creencias y significados.

Según Chen, Lin y Chang (2009) las capacidades de absorción contribuyen a la generación de habilidades de aprendizaje organizacional por medio de la adquisición, asimilación, transformación y explotación de conocimiento obtenido por fuentes externas de información. Sin embargo, es importante mencionar que el proceso de aprendizaje organizacional no es automático: para que ocurra es necesario generar condiciones organizacionales que permitan la difusión y gestión del conocimiento (Alegre, PlaBarber, Chiva, \& Villar, 2012).

El concepto de capacidades dentro de una organización fue definido por Richardson (1972) como la acumulación de conocimiento experiencial y habilidades que generan ventajas competitivas. Esta definición conceptualiza las capacidades desde un enfoque evolutivo y experiencial del conocimiento.

\subsection{Capacidad de Absorción.}

En los últimos años, el estudio de las capacidades dinámicas ha tomado relevancia debido a que permite entender cómo las empresas reconfiguran sus recursos y competencias para ajustarlo a las nuevas condiciones de entorno (Weerawardena, Mort, Liesch, \& Knight, 2007). De esta manera, las capacidades dinámicas son definidas como la combinación de recursos difíciles de imitar que permiten reconfigurar los recursos de la organización ante la dinámica del mercado generando ventajas competitivas de manera sostenible (Açıkdilli \& Yaşar, 2013; Eisenhardt \& Martin, 2000).

La Capacidad de Absorción (CA) es uno de los temas más estudiados en el enfoque evolutivo de la administración estratégica. Es considerada una de las dimensiones de las capacidades dinámicas que estudia la gestión del conocimiento por medio de la capacidad de incorporación de conocimiento intra e inter organizacional (Camison \& Villar-López, 2012). Por ello, la CA ha sido incluida en modelos que buscan explicar los determinantes de las diferentes dimensiones del desempeño empresarial (Neely \& Hii, 1998).

Las primeras definiciones de CA asumen la importancia del conocimiento previo para la adopción de un nuevo conocimiento por medio de un proceso de asociación. Cohen y Levinthal (1989) definieron por primera vez a la CA como la habilidad de las firmas para reconocer el valor de nueva información externa, asimilarla y aplicarla para fines comerciales. Más adelante, Mowery y Oxley (1995) la definen como un amplio conjunto de habilidades necesarias para tratar el componente tácito de los conocimientos transferidos y la necesidad de la modificación de ese conocimiento importado para las necesidades de la empresa. Kim (1997) define a la CA como la capacidad que permite aprender y resolver problemas para la empresa. 
Las definiciones anteriores indican implícitamente la necesidad de una transformación del conocimiento externo para que sea útil y pueda ser explotado con fines comerciales. Por ello, Zahra y George (2002) integran el proceso de aprendizaje por fuentes externas y lo dividen en cuatro dimensiones: (a) adquisición, (b) asimilación, (c) transformación y (d) explotación, que permiten conocer la capacidad potencial y real de las empresas en adoptar un conocimiento obtenido por fuentes externas. El avance de la CA por medio de estudios empíricos se debió a la operacionalización de los constructos por medio de un cuestionario de percepción que permite medir las cuatro dimensiones de CA.

La CA ha sido operacionalizada no solamente por medio de factores sino por medio de proxies relacionados a I+D y a patentes (Koçoglu, Akgün \& Keskin, 2015). En las medidas de CA basadas en I+D han sido adaptadas para identificar el efecto del tamaño y del sector de la empresa. Entre las formas de medir la CA encontramos las siguientes: (a) intensidad en $\mathrm{I}+\mathrm{D}$, definida como el gasto en $\mathrm{I}+\mathrm{D}$ dividido por las ventas totales (Cohen \& Levinthal,1990; Tsai ,2001; Belderbos, Carree, Diederen, Lokshin \& Veugelers, 2004; Lin, Wu, Chang, Wang \& Lee, 2012; Oh, 2016; Fernald, Pennings, Van den Bosch, Commandeur, \& Claassen, 2017); (b) número de patentes (Mowery, Oxley \& Silverman, 1996; Ahuja \& Katila,2001); (c) cantidad de publicaciones académicas (Cockburn \& Henderson, 2003); (d) variables dicotómicas vinculadas con las actividades de I+D (Oltra \& Flor; 2003; Del CarpioGallegos \& Miralles-Torner, 2018) entre las más utilizadas.

Estas medidas de CA basadas en el resultado de la I+D han sido criticadas ya que no capturan el proceso de la gestión del conocimiento externo, por lo que también se utilizan medidas multidimensionales de la CA (Zahra \& George, 2002). Sin embargo, es necesario tener en cuenta que la inversión en I+D es una condición necesaria para la creación de capacidad de absorción (Tsai, 2001). El aumento de la capacidad de absorción a través de inversiones en esfuerzos internos de I+D aumenta la eficacia de la información entrante (René, Carree, Diederenc, Lokshin \& Veugelers, 2004). Henderson y Cockburn (1996) sostienen que las inversiones en I+D pueden mejorar la capacidad de una empresa para reconocer, asimilar y explotar nueva información externa.

Las inversiones en I+D no solo contribuyen a la generación de innovación, sino que también al desarrollo de capacidades y competencias, que no suelen ser valoradas como resultado del proceso innovador (Oh, 2017). Con ello, la innovación no solo permite solucionar problemas y aprovechar oportunidades, sino que también genera conocimiento que puede ser transmitido dentro de la empresa y fuera de ella por medio de la interacción con los miembros externos de la red de negocios (Lin, Wu, Chang, Wang, \& Lee, 2012).

En consecuencia, el uso de una medida de CA basado en la I+D ha sido ampliamente utilizada para el estudio de la innovación en diferentes unidades de análisis: (a) en el estudio de países (Mowery \& Oxley, 1995; Keller,1996; Liu \& White,1997; Pérez \& Toro, 2018); (b) por diferentes tipos de industrias (Del Carpio Gallegos \& Miralles Torner, 2018; Stock, Greis \& Fischer, 2001); (c) a nivel interorganizacional (Backmann, Hoegl \& Cordery, 2015; Lane \& Lubatkin, 1998) y (d) a nivel organizacional (Cohen \& Levinthal, 1990).

En la práctica las inversiones en $\mathrm{I}+\mathrm{D}$ de una empresa se consideran un proxy válido para la $\mathrm{CA}$, en donde existen diferentes argumentos que justifican su uso como se observa en la Tabla 1. La CA basada en inversión en I+D se constituye en un vínculo entre el conocimiento externo y el logro de altos niveles de desempeño de la innovación (Tsai, 2008; Hagedoorn, 2012; Catozzella, 2014). Oltra y Flor (2003) afirman que la posesión de CA, expresada desde un enfoque sistemático de la $\mathrm{I}+\mathrm{D}$, manifiesta una influencia positiva en la producción de innovación. Por lo tanto, las empresas con mayores inversiones en $\mathrm{I}+\mathrm{D}$, tendrán una mayor $\mathrm{CA}$; esto a su vez le permitirá generar resultados innovadores más exitosos en el futuro y exhibirá un desempeño futuro más alto $(\mathrm{Oh}, 2017)$. 
Tabla 1. Capacidad de Absorción (CA): Conceptualización y Operacionalización.

\begin{tabular}{|c|c|c|c|c|c|}
\hline $\mathbf{N}^{0}$. & Autores & Nombre del artículo & $\begin{array}{c}\text { Indicador } \\
\text { CA }\end{array}$ & Argumentos & Metodología \\
\hline 1 & $\begin{array}{l}\text { Stock, Greis \& } \\
\text { Fischer (2001) }\end{array}$ & $\begin{array}{l}\text { Capacidad de } \\
\text { absorción y desarrollo } \\
\text { de nuevos productos. }\end{array}$ & $\begin{array}{c}\text { Gasto en } \\
\text { I+D/VENTAS }\end{array}$ & $\begin{array}{l}\text { Cohen y Levinthal }(1989,1990) \\
\text { encontraron una relación positiva entre } \\
\text { I\&D y aprendizaje. } \\
\text { Kamien y Schwartz (1982) revisaron } \\
\text { un número de estudios que indican una } \\
\text { fuerte correlación positiva entre la } \\
\text { intensidad de I+D y las medidas de } \\
\text { resultados de innovación. }\end{array}$ & $\begin{array}{l}\text { Regresión de } \\
\text { Mínimos } \\
\text { Cuadrados }\end{array}$ \\
\hline 2 & Tsai (2001) & $\begin{array}{l}\text { Transferencia de } \\
\text { conocimientos en } \\
\text { redes } \\
\text { intraorganizacionales: } \\
\text { efectos de la posición } \\
\text { de la red y de la } \\
\text { capacidad abortiva en } \\
\text { la innovación y el } \\
\text { desempeño de la } \\
\text { unidad de negocio. }\end{array}$ & $\begin{array}{c}\text { Gasto en } \\
\text { I+D/VENTAS }\end{array}$ & $\begin{array}{l}\text { La capacidad de absorción de una } \\
\text { unidad organizativa para el aprendizaje } \\
\text { depende de su dotación de capacidades } \\
\text { relevantes basadas en tecnología } \\
\text { (Mowery et al., 1996). } \\
\text { La inversión en I+D es una condición } \\
\text { necesaria para la creación de capacidad } \\
\text { de absorción. Como sugirieron Cohen } \\
\text { y Levinthal (1989), la capacidad de } \\
\text { utilizar el conocimiento externo es a } \\
\text { menudo un subproducto de la } \\
\text { inversión en I+D. Las unidades } \\
\text { organizativas con un alto nivel de } \\
\text { capacidad de absorción invierten más } \\
\text { en su propia I+D y tienen la capacidad } \\
\text { de producir más innovaciones. }\end{array}$ & $\begin{array}{l}\text { Regresión } \\
\text { jerárquica }\end{array}$ \\
\hline 3 & $\begin{array}{l}\text { Lin, Wu, } \\
\text { Chang, Wang } \\
\text { \& Lee }(2012)\end{array}$ & \begin{tabular}{lcc}
\multicolumn{2}{l}{ El desempeño de la } \\
innovación de & la \\
alianza de & $\mathrm{I}+\mathrm{D}:$ & la \\
perspectiva & de & la \\
capacidad & & de \\
absorción. & &
\end{tabular} & $\begin{array}{c}\text { Gasto en } \\
\text { I+D/VENTAS }\end{array}$ & $\begin{array}{l}\text { Una mayor intensidad indica la mayor } \\
\text { capacidad tecnológica interna de la } \\
\text { firma. Una empresa con un alto nivel } \\
\text { de intensidad de I+D es más capaz de } \\
\text { explotar vínculos con otras empresas y } \\
\text { crear conocimiento. }\end{array}$ & $\begin{array}{l}\text { Regresión } \\
\text { Binomial } \\
\text { Negativa }\end{array}$ \\
\hline 4 & Oh (2017) & 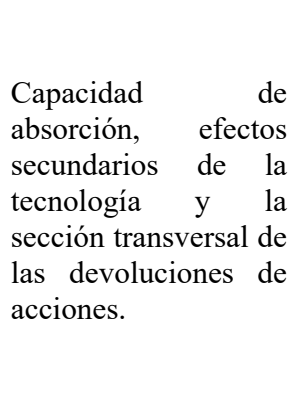 & $\begin{array}{l}\text { Capital I\&D / } \\
\text { Ventas de la } \\
\text { firma }\end{array}$ & $\begin{array}{l}\text { Según Chan et al. (2001) se calcula } \\
\text { como un gasto acumulado de } 5 \text { años en } \\
\text { I+D suponiendo una tasa de } \\
\text { depreciación anual del } 20 \% \text {. } \\
\text { Las inversiones en I+D pueden } \\
\text { mejorar la capacidad de una empresa } \\
\text { para reconocer, asimilar y explotar } \\
\text { nueva información externa, a saber, } \\
\text { "capacidad de absorción" (por } \\
\text { ejemplo, Cohen \& Levinthal, 1989, } \\
\text { 1990; Henderson \& Cockburn, 1996). }\end{array}$ & $\begin{array}{l}\text { Regresión } \\
\text { Fama- } \\
\text { McBeth }\end{array}$ \\
\hline 5 & $\begin{array}{l}\text { Fernald, } \\
\text { Pennings, Van } \\
\text { den Bosch, } \\
\text { Commandeur } \\
\text { \& Claassen } \\
\text { (2017) }\end{array}$ & $\begin{array}{l}\text { El papel moderador } \\
\text { de la capacidad de } \\
\text { absorción y los } \\
\text { efectos diferenciales } \\
\text { de las adquisiciones y } \\
\text { alianzas en el } \\
\text { desempeño } \\
\text { innovación de las } \\
\text { grandes empresas } \\
\text { farmacéuticas. }\end{array}$ & $\begin{array}{c}\text { Gasto en } \\
\mathrm{I}+\mathrm{D} / \text { VENTAS }\end{array}$ & $\begin{array}{l}\text { La intensidad en } \mathrm{I}+\mathrm{D} \text { es el indicador } \\
\text { que se usa con mayor frecuencia y } \\
\text { toma como referencia el trabajo de Lin } \\
\text { et al. (2012). }\end{array}$ & $\begin{array}{l}\text { Regresión de } \\
\text { Poisson }\end{array}$ \\
\hline
\end{tabular}

Fuente: Elaboración propia. 


\subsection{Desempeño Innovador.}

Cuando se habla de desempeño innovador, hay que tener en cuenta que éste corresponde a resultados en términos de desempeño de producto, proceso, marketing y organizacional (Alegre, Lapiedra \& Chiva, 2005). Por ello, el estudio del desempeño innovador se orienta a diversos tipos de innovación. Es decir, la innovación como una estrategia empresarial, lleva a solucionar un problema o responder ante una oportunidad específica. Por tanto, la evaluación del resultado de la innovación debe estar vinculada al objetivo de la innovación.

En primera instancia, es necesario diferenciar entre la innovación desde el punto de una estrategia y la innovación como una capacidad organizacional, debido a que una empresa puede lograr una innovación, sin que esto signifique el logro de la capacidad de innovación, dado que la innovación puede ser adoptada por fuentes externas a la empresa.

Pese a las diferencias en los distintos tipos de innovación, estos pueden estar relacionados. De acuerdo con Utterback y Abernathy (1975), los resultados de innovación de productos y de procesos están relacionados, cuando se toma en consideración que la innovación involucra las funciones de las empresas. Desde la perspectiva del aprendizaje organizacional, no solo el desarrollo de la innovación sino también su implementación, aumenta el inventario de conocimiento de la empresa.

Por la complejidad del estudio de la innovación empresarial, sus antecedentes han sido estudiados desde diferentes perspectivas teóricas sin que esto implique el logro de una taxonomía comúnmente aceptada. Las características de la industria, de la empresa y del entorno en donde se desarrolla tienen una influencia no solo en el logro de altos niveles de innovación, sino también en las barreras que inhiben la innovación empresarial. Pese al amplio estudio del tema, el desempeño innovador de las empresas no solo no tiene un marco teórico comúnmente aceptado, sino que las diferentes formas de operacionalización en estudios empíricos han impedido la generalización de los resultados.

Para Sánchez, Juliao y Zuluaga (2013) una de las principales limitaciones en el estudio de la innovación es la dificultad para medir sus resultados. En el caso del estudio de los resultados de innovación de productos, las diferentes formas de operacionalización se basan en la definición propuesta por la OECD (2005a), que determina como productos innovadores a aquellos totalmente nuevos o productos significativamente mejorados (Arias-Pérez, Schafer-Elejalde \& Aristizábal-Botero, 2014).

Para la operacionalización del desempeño innovador se han utilizado medidas de percepción y objetivas. Para las medidas de percepción del desempeño innovador, se han propuesto escalas construidas por ítems que recogen respuestas mediante escalas Likert. Entre algunas medidas de percepción de desempeño innovador están las propuestas por Sok y O’Cass (2011), Alegre, Lapiedra y Chiva (2006), Lee, Kim y Lee (2011). Mientras que, a partir de los criterios de desempeño de innovación del Manual de Oslo, se ha realizado el análisis factorial exploratorio para identificar estructuras factoriales subyacentes (Paula \& Silva, 2018).

Respecto a indicadores objetivos de desempeño innovador, se han utilizado medidas de relacionadas al número de patentes y ventas de productos nuevos o mejorados (Yam, Lo, Tang \& Law, 2011). En otros estudios, la operacionalización del desempeño innovador de productos está definida como una variable dummy en la que se determina la existencia o no de innovación de producto (Fernandes, Lourenço \& Silva, 2014).

\subsection{Pertinencia de la Capacidad de Absorción en el Desempeño Innovador.}

Hoy en día, las empresas de todas las industrias se involucran y abren sus procesos a la innovación (Chesbrough, 2006). La capacidad de innovar progresivamente se ha vuelto más importante a medida que los estudios revelan que las "empresas innovadoras" tienden a tener una mayor rentabilidad, un 
incremento en el valor de mercado, mejores calificaciones crédito y más probabilidades de supervivencia (Czarnitzki \& Kraft, 2004).

Cohen y Levinthal (1989) sostienen que la CA es un recurso que permite integrar el conocimiento externo en el propio conocimiento de la empresa; este proceso contribuye fundamentalmente al desarrollo de su actividad de innovación (Leiponen, 2005). Puesto que las empresas logran ajustar su organización interna a los cambios en su entorno, exploran oportunidades e incluso soluciones y tienden a explotar la innovación para satisfacer sus necesidades (Zahra \& George, 2002).

Varios estudios revelan que una buena CA puede promover que la empresa absorba y digiera el conocimiento, realizar la transferencia de tecnología rápidamente, contribuir al desarrollo de nuevos productos y posteriormente mejorar los resultados de la innovación (Lin \& Cao, 2008). Así mismo, varios autores confirman que la CA favorece de manera directa (Lichtenthaler, 2009) e indirecta (Lane, Koka, \& Pathak, 2006) al desempeño de la innovación.

Por ello, el estudio de la relación ha sido llevada a diferentes contextos. Las industrias de alta tecnología como la farmacéutica han comprobado que la CA es una variable crítica para mejorar el desempeño de innovación (Jeon, Hong, Ohm \& Yang, 2015; Fernald et al., 2017); mismo criterio obtienen las industrias de baja tecnología como la de panadería y manufacturera (Chen, Lin \& Chang, 2009). Además, diferentes estudios muestran que la CA de las pymes es una variable de alto impacto que mejora su desempeño innovador (Scuotto, Del Giudice \& Carayannis, 2017; Zhai et al., 2018), debido a que facilita el proceso de adquisión de conocimiento externo (Palacios-Marqués, Soto-Acosta \& Merigó, 2015).

En definitiva, la CA se ha considerado una base fundamental para el éxito de la innovación (Chang \& Chob, 2008). Assink (2006) sostiene que el desarrollo de innovación no es un esfuerzo de una sola vez, sino que requiere un desarrollo continuo de la CA para mejorar la capacidad general de innovación de las empresas. Por esta razón las empresas están cooperando activamente con el entorno externo para lograr un desempeño de innovación global que asimismo mejore su creación de valor y productividad. Esto, a su vez, conducirá al logro de ventajas competitivas a largo plazo (CepedaCarrión, Cegarra-Navarro \& Jiménez-Jiménez, 2012).

\subsection{Factores limitantes en el desarrollo de la innovación}

La investigación dirigida a comprender las innovaciones exitosas no es un tema reciente y en los últimos años ha tomado relevancia para las organizaciones (Anderson, De Dreu \& Nijstad, 2004; Verhees \& Meulenberg, 2004). Mirow, Hölzle y Gemünden (2007) indican que los factores que dificultan la innovación han generado interés y son estudiados con mayor frecuencia. Es innegable el valor que genera el identificar los factores clave que explican cómo se puede gestionar la innovación; sin embargo, la capacidad para aprender de los fallos y los desvíos es fundamental para el progreso (Pisano, 2006). Lograr la minimización de los obstáculos en la innovación se considera en realidad como una clave para el éxito innovador (Hall \& Martin, 2005).

Por lo tanto, es importante que las empresas tomen en consideración las barreras presentes en el momento de emprender sus esfuerzos en innovación. Las barreras a la innovación son definidas como factores que impiden, retrasan o bloquean completamente la innovación (Mirow, Hoelzle \& Gemuenden, 2008). El descubrimiento de dichas barreras permitirá que las empresas tomen conciencia de las dificultades que tendrán que enfrentar en el compromiso con las actividades de innovación (D’Este, Iammarino, Savona \& von Tunzelmann, 2012).

La literatura relacionada con las barreras de innovación identifica dos líneas de investigación. La primera línea trata de explicar cómo los obstáculos influyen en la propensión e intensidad de la innovación. En esta dirección se analizan los efectos y las causas de las barreras financieras en otras dimensiones como la innovación organizativa (Blanchard, Huiban, Musolesi \& Sevestre, 2012). Así mismo, otros estudios muestran que la falta de financiación restringe las inversiones en planta o 
maquinaria (Bloom \& Van Reenen, 2007; Hubbard, 1998) y reducen la disposición de las pequeñas y medianas empresas manufactureras para desarrollar actividades de I+D (Mancusi \& Vezzulli, 2014).

La segunda línea trata de identificar y analizar los determinantes de las diversas clases de barreras según las características de las empresas. Sin embargo, esta segunda línea de investigación comparte características similares en cuanto a la estructura del análisis y la elección de variables. Una muestra de ello es el enfoque en los gastos de investigación y desarrollo (I+D) como principal insumo para la innovación (De-Oliveira \& Rodil-Marzábal, 2019).

Pellegrino y Savona (2017) identificaron otros factores al menos tan importantes como las limitaciones financieras que explican las fallas de innovación de las empresas. Al analizar los datos a nivel de empresa del Reino Unido (Community Innovation Survey 2002-2010), estos autores encontraron que ciertos factores no financieros, como la estructura de mercado concentrada y la falta de demanda, determinan el desempeño limitado de la innovación de la empresa. En un estudio realizado recientemente, los autores identifican otro factor determinante en la innovación. Luengo-Valderrey y Moso-Díez (2019) señalan que las empresas interesadas en mejorar su actividad innovadora deben ser conscientes de la importancia de la cualificación de los trabajadores y en conjunto la gestión del conocimiento para generar un mejor desempeño de la innovación.

La innovación exitosa depende de que la empresa combine una gama de capacidades, incluida la capacidad para acceder a financiación, comprender las necesidades del mercado, reclutar personal altamente calificado y establecer interacciones efectivas con otros actores (De-Oliveira \& RodilMarzábal, 2019). Las empresas innovadoras necesariamente se ven obligadas a enfrentar la mayoría, si no todos de estos desafíos. Por ello, es imprescindible tener en cuenta estos tipos de obstáculos para un análisis más coherente en el estudio del desempeño innovador.

\section{Hipótesis a evaluar.}

H1: La Capacidad de Absorción medida por inversión (gastos) en I+D influye en el Desempeño Innovador.

H2: La fuente de origen de la I+D influye en el Desempeño Innovador

H3: Las barreras afectan el desempeño innovador

H4: La inversión en innovación tuvo impacto en el desempeño de las empresas

\section{Marco metodológico.}

Desde la perspectiva de la capacidad de absorción, este estudio pretende analizar la relación existente entre la inversión en $\mathrm{I}+\mathrm{D}$ en el desempeño innovador de las empresas ecuatorianas de la industria manufacturera. La investigación se dirige a este sector debido a que es uno de los más importantes para cualquier nación: la contribución del sector manufacturero al PIB real ha aumentado de $14,8 \%$ a $16 \%$ en las dos últimas décadas a nivel mundial. En el Ecuador, la industria de manufactura es el principal sector de generación de valor agregado, genera el 10,7\% del total de empleo, representa el 13,6\% del PIB y contiene el $8 \%$ del total de las empresas del país (Guerra \& Martin, 2017).

En este estudio, se emplean los datos proporcionados por el Instituto Nacional de Estadísticas y Censos (INEC) de la "Encuesta Nacional de Actividades de Ciencia, Tecnología e Innovación" del periodo 2012 - 2014 el cual provee información de 6.275 empresas en 4 sectores económicos de todas las provincias del Ecuador, de los cuales 3.775 corresponden a la industria manufacturera. Teniendo en 
cuenta la información obtenida se realiza un análisis de regresión lineal y múltiple para relacionar el desempeño innovador de la empresa con la capacidad de absorción.

\section{Análisis de resultados.}

Se selecciona un grupo de empresas que cumplen ciertas condiciones de innovación. En primer lugar, identificamos aquellas empresas que habían realizado ventas de productos innovadores, en total 1.439, tomando como fundamento la definición de innovación en producto del Manual de Oslo (OCDE, 2005b). En segundo lugar, considerando el enfoque en el estudio de la capacidad de absorción, a través de la inversión en $\mathrm{I}+\mathrm{D}$, elegimos a las empresas que durante el periodo de estudio realizaron gastos en I+D independientemente de su origen interno o externo, que fueron 818. Por último, analizamos la intensidad de innovación de las compañías y, por lo tanto, seleccionamos aquellas que tenían al menos $1 \%$ de gastos en I+D respecto a su facturación, donde finalmente se obtienen 201 empresas objeto de análisis.

En Ecuador, la inversión en I+D ha permanecido estancada desde el año 2009 debido a la falta de apoyo externo y gubernamental (CEPAL, 2016). En 2014, la inversión I+D a nivel nacional permanece por debajo del $0,5 \%$ respecto al PIB, considerando la inversión mundial de $2,12 \%$ se confirma la baja propensión de inversión en I+D de los países latinoamericanos (Banco Mundial , 2014). Durante el mismo periodo, el 54\% de las empresas encuestadas por el INEC afirman haber realizado inversiones en innovación, de las cuales un 32\% corresponde a las empresas de la industria de manufactura.

Las empresas manufactureras con un grado de innovación significativo provienen en su mayoría de la región Sierra, un $80 \%$ aproximadamente, donde la industria manufacturera de la provincia de Pichincha está representada con 133 empresas, esto es el 60\% del grupo de empresas. Mientras que el $20 \%$ de las empresas son originarias de la región Costa, de las cuales la provincia del Guayas es la de mayor participación con 43 empresas, un 19\% del total, como se muestra en la Tabla 2. Estos resultados corresponden al hecho de que Pichincha y Guayas son consideradas las capitales comerciales del país.

Tabla 2. Distribución de Empresas por provincia.

\begin{tabular}{ccc}
\hline Provincia & Frecuencia & Porcentaje acumulado \\
\hline Azuay & 10 & 4,6 \\
Bolívar & 2 & 5,5 \\
Chimborazo & 2 & 6,6 \\
Guayas & 43 & 26,0 \\
Imbabura & 13 & 31,9 \\
Loja & 1 & 32,3 \\
Manabí & 2 & 33,0 \\
Napo & 1 & 33,5 \\
Pichincha & 133 & 92,9 \\
Tungurahua & 16 & 100,0 \\
Total & 223 & \\
\hline
\end{tabular}

Fuente: Elaboración propia.

Por otro lado, es importante destacar que no hay un sector totalmente dominante en la industria manufacturera respecto al nivel de innovación empleada. Esto es un indicio de que el sector al que 
pertenece una empresa no determina su intensidad de innovación para el caso ecuatoriano. Sin embargo, el mercado local identifica tres actividades de mayor innovación: la actividad de fabricación de sustancias y productos químicos con 39 empresas o un $17 \%$ del total, seguido de la elaboración de productos alimenticios con 26 empresas $(11,6 \%)$ y la fabricación de maquinaria y equipo con 22 empresas (un 10\% aproximadamente), como se muestra en la Tabla 3.

Tabla 3. Distribución de Empresas por Actividad Económica.

\begin{tabular}{|c|c|c|}
\hline Actividad Económica - CIIU & Frecuencia & $\begin{array}{l}\text { Porcentaje } \\
\text { acumulado }\end{array}$ \\
\hline Elaboración de productos alimenticios & 26 & 11,5 \\
\hline Elaboración de bebidas & 3 & 12,7 \\
\hline Fabricación de productos textiles & 2 & 13,7 \\
\hline Fabricación de prendas de vestir & 4 & 15,3 \\
\hline Fabricación de cueros y productos conexos & 4 & 16,9 \\
\hline $\begin{array}{l}\text { Producción de madera y fabricación de productos de madera y } \\
\text { corcho, excepto muebles; fabricación de artículos de paja y }\end{array}$ & 3 & 18,1 \\
\hline Fabricación de papel y de productos de papel & 2 & 19,0 \\
\hline Impresión y reproducción de grabaciones & 20 & 28,0 \\
\hline Fabricación de substancias y productos químicos & 39 & 45,5 \\
\hline $\begin{array}{l}\text { Fabricación de productos farmacéuticos, sustancias químicas } \\
\text { medicinales y productos botánicos de uso farmacéutico }\end{array}$ & 13 & 51,6 \\
\hline Fabricación de productos de caucho y plástico & 18 & 59,7 \\
\hline Fabricación de otros productos minerales no metálicos & 13 & 65,7 \\
\hline Fabricación de metales comunes & 10 & 70,1 \\
\hline $\begin{array}{l}\text { Fabricación de productos elaborados de metal, excepto maquinaria y } \\
\text { equipo }\end{array}$ & 12 & 75,6 \\
\hline Fabricación de productos de informática, electrónica y óptica & 3 & 77,0 \\
\hline Fabricación de equipo eléctrico & 1 & 77,4 \\
\hline Fabricación de maquinaria y equipo & 22 & 87,1 \\
\hline Fabricación de vehículos automotores, remolques y semirremolques & 3 & 88,4 \\
\hline Fabricación de otros tipos de equipos de transporte & 8 & 92,0 \\
\hline Fabricación de muebles & 4 & 93,9 \\
\hline Otras industrias manufactureras & 8 & 97,5 \\
\hline Reparación e instalación de maquinaria y equipo & 6 & 100,0 \\
\hline Total & 223 & \\
\hline
\end{tabular}

Fuente: Elaboración propia.

La procedencia del capital es un factor importante a considerar por las empresas en economías en desarrollo. En Ecuador, la mayor parte del capital de las empresas es de origen nacional: tan solo un $2,77 \%$ corresponde a capital extranjero. Mismo panorama reflejan las empresas innovadoras objeto de este estudio, cuyo capital producto de inversión extranjera es solo el 1,37\%. La inversión extranjera en 
el país ha permanecido baja como resultado del entorno regulatorio inestable y el débil estado de derecho (CIA, 2019).

El flujo de capital principalmente interno puede ser un factor que desincentiva los esfuerzos de innovación, debido a que dicho capital es cuidadosa y tradicionalmente administrado. Las empresas manufactureras enfrentan además el desafío de ser un sector poco atractivo para la Inversión Extranjera Directa, debido a que para el año 2013 solo el 1\% fue destinado a este sector, cifra que se ha incrementado al 7\% sin ganar mayor representatividad para el 2018.

A pesar de ello, la industria manufacturera ecuatoriana, a lo largo del tiempo, no ha sido indiferente a la innovación. Existen empresas de gran trayectoria con ventas innovadoras y otras de reciente constitución. La empresa más antigua tiene 74 años a la fecha de cohorte de la encuesta y presenta una alta proporción en ventas innovadoras. Un 50\% de las empresas con ventas innovadoras fueron creadas a partir de año 2000; sin embargo, el comportamiento respecto a las ventas en innovación no difiere en gran medida considerando las edades, debido a que existen empresas nuevas con bajo nivel de ventas en productos innovadores y empresas antiguas con alto nivel de ventas innovadoras.

Las empresas innovadoras en el Ecuador tienen un promedio de facturación de 5,5 millones de dólares. Un 39\% de las ventas totales corresponden a productos innovadores. Sin embargo, las empresas en promedio destinan una cantidad mínima de $5 \%$ a la inversión $\mathrm{I}+\mathrm{D}$, un gasto promedio menor a 0,4 millones, de los cuales la inversión en I+D interna es más intensiva con un $82 \%$. Las cifras mencionadas reflejan la subvaloración que tienen las empresas respecto a la inversión en $\mathrm{I}+\mathrm{D}$ o la Capacidad de Absorción que es la variable de estudio.

Para probar las hipótesis planteadas inicialmente, se especifican varios modelos de regresión que analizan la relación entre la capacidad de absorción y el desempeño innovador de las empresas, así como el efecto de las barreras de innovación. En primer lugar, se analiza la existencia de multicolinealidad en los modelos para garantizar que no haya problema en los modelos; se calcularon los factores de inflación de varianza (VIF) para las variables. Los valores del VIF están por debajo del nivel crítico de 10, lo cual significa que la multicolinealidad no contamina los resultados (O'Brian, 2007). Por lo tanto, no se planteó ninguna preocupación seria sobre la multicolinealidad.

Tabla 4. Resultados del Análisis de Regresión.

\begin{tabular}{llll}
\hline & Modelos & \multicolumn{2}{l}{ Variable Dependiente :Ventas Innovadoras } \\
\cline { 2 - 4 } Variable & 1 & 2 & 3 \\
\hline Intercepto & 185686,93 & 196789,916 & 389948,549 \\
CA (I+D) & $2,27^{*}$ & & \\
I+D interna & & $2,25^{*}$ & $2,84^{* *}$ \\
I+D externo & & 0,329 & 1,127 \\
Tamaño & $26988,94^{* * *}$ & $27037,97^{* * *}$ & $26415,68^{* * *}$ \\
Barrera de fondos & & & $\left(-674863,26^{* *}\right)$ \\
Barrera de financiamiento & & & 444396,242 \\
Barrera de costo de innovación & & 0,669 & 4582,681 \\
$R^{2}$ & 0,669 & & 0,677 \\
\hline
\end{tabular}

${ }^{*} \mathrm{p}<.1,{ }^{* *} \mathrm{p}<.05,{ }^{* * *} \mathrm{p}<.01$ significancia de la variable

Fuente: Elaboración propia.

Para analizar la influencia de la capacidad de absorción sobre el desempeño innovador se estiman tres modelos. El primer modelo consiste en un análisis de regresión lineal para evaluar la veracidad de 
la hipótesis principal. El modelo 2 corresponde a un análisis de regresión múltiple donde se desagrega la variable de inversión $\mathrm{I}+\mathrm{D}$ desde su origen interno o externo. Posteriormente el modelo 3 analiza el efecto de las barreras de innovación basándose en la estructura del segundo modelo.

Con el objetivo de probar la H1, la hipótesis principal, se relacionan las variables correspondientes a las ventas innovadoras y la capacidad de absorción, tal como lo muestra la Tabla 3. Se encontró que la CA operacionalizada a traves de la inversión en $\mathrm{I}+\mathrm{D}$ tiene un efecto significativo y positivo en las ventas innovadoras.

Por medio de la estimación del modelo 2, donde la variable que contiene la inversión en I+D se desagrega, se encontró que la $\mathrm{I}+\mathrm{D}$ interna tiene un efecto significativo en el desempeño innovador de las empresas, mientras que la I+D externa carece de significancia. Este resultado confirma la segunda hipótesis planteada, relativa a la importancia que tiene el origen de la inversión en I+D en el desempeño innovador.

Bajo la misma estructura se incluyó el efecto de las barreras innovadoras en la relación entre la capacidad de absorción y el desempeño innovador de las empresas ecuatorianas. La barrera de innovación enfocada en el costo fue seleccionada para el análisis debido a que es la barrera señalada como la más importante por las empresas analizadas. Esta barrera fue operacionalizada por medio de tres variables dummy que representan los obstáculos que las empresas enfrentan respecto a la disponibilidad de fondos dentro de la empresa, la falta de financiación de fuentes de externas y los costos altos de producto de las actividades de innovación.

Por último, para evaluar la H4 donde se relaciona el impacto de la inversión en innovación en el desempeño de las empresas, se utiliza un Análisis de Componente Principal (ACP). Por medio de este procedimiento se extraen las variables que aportan mayor información y se compactan en componentes de mayor relevancia.

Tabla 5. Resultados del ACP en el impacto de las innovaciones.

\begin{tabular}{|c|c|c|c|}
\hline & \multicolumn{2}{|c|}{ Componente } \\
\hline & & 1 & 2 \\
\hline \multirow{6}{*}{$\begin{array}{l}\text { C1: } \\
\text { Desempeño de } \\
\text { mercado }\end{array}$} & $\begin{array}{l}\text { Impacto del "Aumentó la variedad de bienes o servicios" } \\
\text { debido a la introducción de innovaciones }\end{array}$ & 0,780 & 0,256 \\
\hline & $\begin{array}{l}\text { Impacto del "Reemplazó los productos o procesos } \\
\text { desactualizados" debido a la introducción de innovaciones }\end{array}$ & 0,522 & 0,332 \\
\hline & $\begin{array}{l}\text { Impacto del "Ingresó a nuevos mercados" debido a la } \\
\text { introducción de innovaciones }\end{array}$ & 0,795 & 0,145 \\
\hline & $\begin{array}{l}\text { Impacto del "Incremento de la participación de mercado" } \\
\text { debido a la introducción de innovaciones }\end{array}$ & 0.850 & -0.055 \\
\hline & $\begin{array}{l}\text { Impacto del "Mejoró la calidad de bienes o servicios" } \\
\text { debido a la introducción de innovaciones }\end{array}$ & 0,709 & 0,265 \\
\hline & $\begin{array}{l}\text { Impacto del "Mejoró la flexibilidad para producir bienes o } \\
\text { servicios" debido a la introducción de innovaciones }\end{array}$ & 0,620 & 0,468 \\
\hline \multirow{3}{*}{$\begin{array}{l}\text { C2: } \\
\text { Desempeño de } \\
\text { procesos }\end{array}$} & $\begin{array}{l}\text { Impacto del "Aumentó la capacidad para producir bienes } \\
\text { servicios" debido a la introducción de innovaciones }\end{array}$ & 0,338 & 0,738 \\
\hline & $\begin{array}{l}\text { Impacto del "Redujo los costos de producción por unidad de } \\
\text { producción" debido a la introducción de innovaciones }\end{array}$ & 0,240 & 0,895 \\
\hline & $\begin{array}{l}\text { Impacto del "Redujo los costos de materiales y energía por } \\
\text { unidad de producción" debido a la introducción de } \\
\text { innovaciones }\end{array}$ & 0,017 & 0,876 \\
\hline
\end{tabular}

Fuente: Elaboración propia. 
Dentro del impacto de la inversión de innovación en empresas ecuatorianas, el análisis revela una mejora en eficiencia empresarial a nivel de mercado y de procesos. Por lo tanto, la Capacidad de Absorción o inversión en I+D, como promotor de la innovación crea un ambiente para generar ventajas competitivas sostenibles, mediante la capacidad de incorporar conocimiento intra e interorganizacional que permite a las empresas reconfigurarse ante la dinámica del mercado y la incorporación de nuevas tecnologías en los procesos de la industria manufacturera.

\section{Conclusiones.}

Este estudio revela interesantes resultados que contribuyen al entendimiento del alcance y relevancia de la innovación en las empresas de la industria manufacturera ecuatoriana. En primera instancia, se valida el efecto positivo que ejerce la CA de la empresa sobre el desempeño innovador, tal como la teoría sugiere.

Las empresas ecuatorianas cada vez más se involucran en actividades para desarrollar innovación. Entre las principales motivaciones se encuentran llegar a ser empresas proactivas para identificar apropiadamente oportunidades en el Mercado. Así mismo, las compañías consideran a la innovación una herramienta no sólo para hacer frente a las amenazas de la competencia sino también para no perder participación en su mercado. Por ello, los gastos en I+D para aprovechar dicha información del entorno se han convertido en una inversión imprescindible para el proceso de innovación.

Esta investigación confirma que la actividad de I+D desarrollada por la empresa genera efectos diferentes si se considera la fuente de la información. Para la empresa ecuatoriana innovadora cada dólar que invierte en I+D externa, $18,75 \$$ son destinados a la inversión en $\mathrm{I}+\mathrm{D}$ interna. En las empresas de la industria manufacturera la concurrencia en actividades de I+D interna generará resultados más innovadores que la I+D externa, esto no implica que estas empresas no han tenido información de fuentes externas, más bien, esto conlleva al entendimiento que para las empresas ecuatorianas es necesario que exista un proceso interno que configure la información interna y externa para que se genere la innovación.

Para un análisis más profundo, se examinan factores del entorno que obstaculizan la innovación. La barrera respecto a la disponibilidad de fondos es la más significativa, este resultado es coherente con el hecho de que la principal fuente de financiación de las empresas analizadas es de origen nacional o interno, por lo tanto, si esta fuente es limitada reduce la posibilidad de inversiones en I+D y por ende futuros desarrollos en innovación.

Dentro de la industria manufacturera ecuatoriana, una empresa que invierte al menos el $1 \%$ de sus ventas totales en I+D y no percibe ninguna barrera en su entorno podría tener una mejora del 4,03\% en su facturación. Sin embargo, muchas empresas no pueden disfrutar de este beneficio debido a que la barrera de disponibilidad de fondos genera un impacto negativo en las ventas innovadoras y es muy probable que éstas sean las que menos invierten en $\mathrm{I}+\mathrm{D}$ debido a que no obtienen el retorno esperado.

Estos hallazgos revelan la importancia de la inversión en I+D para el crecimiento financiero de las empresas; así mismo, hace énfasis en la necesidad de crear estrategias que le permitan superar aquellas barreras que impiden su innovación y la generación de ventajas competitivas.

Por medio del análisis del impacto de la innovación se descubren los efectos transversales de las actividades de $\mathrm{I}+\mathrm{D}$. Los esfuerzos de innovación no solamente generan una mejora en el rendimiento financiero, sino que conlleva la eficacia de otros resultados no financieros como es el desempeño de mercado y de procesos. La innovación ha permitido a las empresas incrementar su participación en el mercado como resultado de ofertar productos actualizados con mayor variedad, calidad y con valor agregado. Con respecto a los procesos, el impacto de la innovación ha permitido a las empresas 
manufactureras ecuatorianas incrementar sus capacidades de producción a la par de una reducción de costos tanto en materia prima como en la producción debido a la optimización de recursos.

Como se ha visto a lo largo de la investigación, la innovación es un proceso de gran valor con influencia contundente en el desempeño empresarial tanto a nivel de ingresos como en el proceso de aprendizaje. La Capacidad de Absorción ha demostrado ser una competencia organizacional significativa para el aprovechamiento del conocimiento externo que en conjunto al desarrollo interno de la empresa mejora los procesos y las características de los productos, habilidad que permite adaptarse a las condiciones cambiantes del entorno. Por lo tanto, los gastos en $\mathrm{I}+\mathrm{D}$ deben verse como una condición necesaria para que las empresas manufactureras ecuatorianas logren tomar mejores decisiones, superar obstáculos y alcanzar mayores niveles de competitividad a nivel local e internacional.

\section{Futuras Investigaciones.}

En este estudio se analizan las empresas manufactureras que reportan ventas innovadoras a partir del desarrollo innovador de productos con inversiones en I+D activas dentro del periodo de análisis 20122014. Este sector es rico en diversidad tanto en actividades, tamaños, intensidad tecnológica como en edad o ubicación geográfica. Esta diversidad genera una gran limitación en la investigación: los datos proporcionados por el INEC dificultan un estudio particular que permita hallazgos más profundos y utilizables para un determinado de grupo de empresas.

Otra limitación presente es no poder contar con datos cuantitativos completos para cada período de análisis, por lo tanto, se validaron los modelos en base a datos de corte transversal que comprendían los tres años de estudio. A pesar de estas limitaciones, este estudio ha sido útil para revelar hallazgos interesantes y generar un avance en literatura sobre el efecto de la Capacidad de Absorción en Ecuador.

\section{Referencias}

Açıkdilli, G., \& Yaşar, D. (2013). Dynamic capabilities and entrepreneurial orientation in the new product development. International Journal of Business and Social Science, 4(11), 145-150.

Ahmed, P. K., \& Shepherd, C. D. (2012). Administración de la Innovación. México: Pearson Editorial.

Ahuja, G., \& Katila, R. (2001). Technological acquisitions and the innovation performance of acquiring firms: a longitudinal study. Strategic Management Journal, 22(3), 197-220.

Alegre, J., Lapiedra, R., \& Chiva, R. (2005). Propuesta y validación de una escala de medida del desempeño innovador de la empresa. Documento presentado en el Congreso Nacional de la $A C E D E$. La Laguna, España.

Alegre, J., Lapiedra, R., \& Chiva, R. (2006). A measurement scale for product innovation performance. European Journal of Innovation Management, 9(4), 333-346.

Alegre, J., Pla-Barber, J., Chiva, R., \& Villar, C. (2012). Organisational learning capability, product innovation performance and export intensity. Technology Analysis \& Strategic Management, 24(5), 511-526.

Anderson, N., De Dreu, C., \& Nijstad, B. (2004). The routinization of innovation research: a constructively critical review of the state-of-the-science. Journal of Organizational Behavior, 25(2), 147-173. 
Arias-Pérez, J., Schafer-Elejalde, G., \& Aristizábal-Botero, C. (2014). Relación entre desempeño innovador y madurez de capacidades de conocimiento y competencia. Entramado, 10(1), 8295.

Arundel, A. (1997). Enterprise strategies and barriers to innovation. Innovation Measurement and Policies, 50, 101-108.

Assink, M. (2006). Inhibitors of disruptive innovation capability: A conceptual model. European Journal of Innovation Management, 9(2), 215-233.

Backmann, J., Hoegl, M., \& Cordery, J. L. (2015). Soaking It Up: Absorptive Capacity in Interorganizational New Product Development Teams. Journal of Product Innovation Management, 32(6), 861-877.

Banco Mundial (2014). Databank. Recuperado de https://datos.bancomundial.org/indicador/GB.XPD.RSDV.GD.ZS?locations=EC$1 \mathrm{~W} \&$ name_desc $=$ false

Barney, J., Della, V., Sciarelli, M., \& Arikan, A. (2012). The role of resource-based theory in strategic management studies: Managerial implications and hints for research. En G. Battista, Handbook of research on competitive strategy (págs. 109-146). Cheltenham: Edward Elgar Publishing.

Belderbos, R., Carree, M., Diederen, B., Lokshin, B., \& Veugelers, R. (2004). Heterogeneity in R\&D cooperation strategies. International Journal of Industrial Organization, 22(8-9), 1237-1263.

Blanchard, P., Huiban, J.-P., Musolesi, A., \& Sevestre, P. (2012). Where there is a will, there is a way? Assessing the impact of obstacles to innovation. Industrial and Corporate Change, 22(3), 679710 .

Bloom, N., \& Van Reenen, J. (2007). Measuring and Explaining Management Practices Across Firms and Countries. The Quarterly Journal of Economics, 122(4), 1351-1408.

Camison, C., \& Villar-López, A. (2012). On how firms located in an industrial district profit from knowledge spillovers: Adoption of an organic structure and innovation capabilities. British Journal of Management, 23(3), 361-382.

Casillas, J., Moreno, A., Acebo, F., Gallego, M., \& Ramos, E. (2009). An integrative model of the role of knowledge in the internationalization process. Journal of World Business, 44(3), 311-322.

Castañeda, D. (2015). Condiciones para el aprendizaje organizacional. Estudios Gerenciales, 31(134), 62-67.

Catozzella, V. (2014). The catalysing role of in-house R\&D in fostering complementarity among innovative inputs. Industry and Innovation, 21(3), 179-96.

CEPAL (2016). Ciencia, tecnología e innovación en la economía digital. Segunda Reunión de la Conferencia de Ciencia, Innovación y TIC de la CEPAL (pp. 19-22). San José.

Cepeda-Carrión, G., Cegarra-Navarro, J.G., \& Jiménez-Jiménez, D. (2012). The effect of absorptive capacity on innovativeness: Context and information systems capability as catalysts. British Journal of Management, 23(1), 110-129.

Chan, L.K., Lakonishok, J., \& Sougiannis, T. (2001). The Stock Market Valuation of Research and Development Expenditures. The Journal of Finance, 56, 2431-2456.

Chang, D.R., \& Chob, H. (2008). Organizational memory influences new product success. Journal of Business Research, 61(1), 13-23. 
Chen, Y.-S., Lin, M.-J. J., \& Chang, C.-H. (2009). The positive effects of relationship learning and absorptive capacity on innovation performance and competitive advantage in industrial markets. Industrial Marketing Management, 38(2), 152-158.

Chesbrough, H. (2006). Open Business Models: How to Thrive in the New Innovation Landscape. Boston, MA: Harvard Business Press.

Chiva, R., \& Alegre, J. (2005). Organizational learning and organizational knowledge toward the integration of two approaches. Management Learning, 31(6), 49-68.

CIA. (26 de 06 de 2019). Central Inteligence Agency. Recuperado de The World Factbook: https://www.cia.gov/library/publications/the-world-factbook/geos/ec.html

Cockburn, I.M., \& Henderson, R.M. (2003). Absorptive Capacity, Coauthoring Behavior, and the Organization of Research in Drug Discovery. The Journal of Industrial Economics, 46(2), 157182.

Cohen, W.M., \& Levinthal, D.A. (1990). Absorptive capacity: A new perspective on learning and innovation. Administrafive Science Quarterly, 35, 128-152.

Cohen, W., \& Levinthal, D. (1989). Innovation and learning: The two faces of R\&D. Economic Journal, 99(397), 569-596.

Czarnitzki, D., \& Kraft, K. (2004). Control Management and Innovative Activity. Review of Industrial Organization, 24(1), 1-24.

D’Este, P., Iammarino, S., Savona, M., \& von Tunzelmann, N. (2012). What hampers innovation? Revealed barriers versus deterring barriers. Research Policy, 41(2), 482-488.

Damanpour, F., Szabat, K., \& Evan, W. (1989). The relationship between types of innovation and organizational performance. Journal of Management Studies, 26(6), 587-601.

Del Carpio, J.F., \& Miralles, F. (2018). Absorptive capacity and innovation in low-tech companies in emerging economies. Journal of Technology Management \& Innovation, 13(2), 3-11.

De-Oliveira, F., \& Rodil-Marzábal, Ó. (2019). Structural characteristics and organizational determinants as obstacles to innovation in small developing countries. Technological Forecasting and Social Change, 140(C), 306-314.

Eisenhardt, K., \& Martin, J. (2000). Dinamic capabilities: What are they? Strategic Management Journal, 21(10), 1105-1121.

Fernald, K.D., Pennings, H.P., Van den Bosch, J.F., Commandeur, H.R., \& Claassen, E. (2017). The moderating role of absorptive capacity and the differential effects of acquisitions and alliances on Big Pharma firms' innovation performance. PLOS ONE, 12(2), 1-22.

Fernandes, A., Lourenço, L., \& Silva, M. (2014). Influence of Quality Management on the Innovative Performance. Revista Brasileira de Gestao de Negocios, 16(53), 575-593.

Govindarajan, V., \& Trimble, C. (2013). Innovación Inversa. Bogotá: Carvajal Educación S.A.S.

Guerra, F.J., \& Martin-Montaner, J.A. (2017). Desarrollo Histórico de la Industria Manufacturera Ecuatoriana y su matriz de exportación. Revista Publicando, 4(2), 504-521.

Hagedoorn, W. N. (2012). Is there complementarity or substitutability between internal and external R\&D strategies? Research Policy, 41(6), 1072-1083. 
Hall, J., \& Martin, M. (2005). Disruptive technologies, stakeholders and the innovation value-added chain: a framework for evaluating radical technology development . R\&D Management, 35(3), 273-284.

Henderson, R., \& Cockburn, I. (1996). Scale, scope, and spillovers: the determinants of research productivity in drug discovery. RAND Journal of Economics, 27, 32-59.

Holzl, W., \& Janger, J. (2013). Does the analysis of innovation barriers perceived by high growth firms provide information on innovation policy priorities? Technological Forecasting \& Social Change, 80, 1450-1468.

Hubbard, R.G. (1998). Capital-Market Imperfections and Investment. Journal of Economic Literature, $36(1), 193-225$.

Jeon, J., Hong, S., Ohm, J., \& Yang, T. (2015). Causal Relationships among Technology Acquisition, Absorptive Capacity, and Innovation Performance: Evidence from the Pharmaceutical Industry. PLOS ONE, 10(7), 1-14.

Kamien, M., \& Schwartz, N. (1982). Market Structure and Innovation. Cambridge: Cambridge University Press.

Keller, W. (1996). Absorptive capacity: On the creation and acquisition of technology in development. Journal of Developmental Economics, 49, 199-210.

Kim, D. (1997). From imitation to innovation: The dynamics of Korea's technological learning. Harvard Business School Press.

Koçoglu, I., Akgün, A.E., \& Keskin, H. (2015). The differential relationship between absorptive capacity and product innovativeness: a theoretically derived framework. International Business Research, 8(7), 108-120.

Lane, P.J., \& Lubatkin, M. (1998). Relative absorptive capacity and interorganizational learning. Strategic Management Journal, 19, 461-477.

Lane, P. J., Koka, B.R., \& Pathak, S. (2006). The Reification of Absorptive Capacity: A Critical Review and Rejuvenation of the Construct. Academy of Management Review, 31(4), 833-863.

Lee, Y., Kim, S., \& Lee, H. (2011). The impact of service R\&D on the performance of Korean information communication technology small and medium enterprises. Journal of Engineering and Technology Management, 28(1), 77-92.

Leiponen, A. (2005). Skills and innovation. International Journal of Industrial Organization, 23(5-6), 303-323.

Lichtenthaler, U. (2009). Absorptive capacity, environmental turbulence, and the complementarity of organizational learning processes. Academy of Management Journal, 52(4), 822-846.

Lin, C., Wu, Y.-J., Chang, C., Wang, W., \& Lee, C.-Y. (2012). The alliance innovation performance of R\&D alliances: the absorptive capacity perspective. Technovation, 32(5), 282-292.

Lin, J., \& Cao, J. (2008). Study on the knowledge-sharing mechanism of knowledge management of cluster enterprises. Science \& Technology Progress and Policy, 4, 161-164.

Liu, X., \& White, R.S. (1997). The relative contributions of foreign technology and domestic inputs to innovation in Chinese manufacturing industries. Technovation, 17, 119-125. 
Luengo-Valderrey, M.J., \& Moso-Díez, M. (2019). Interaction Between Knowledge Management Activities, Innovation Barriers and Innovation Performance: Spanish High and Medium Technology Firms. Journal of the Knowledge Economy, 10(1), 298-317.

Madrid-Guijarro, A., García, D., \& Van Auken, H. (2009). Barriers to Innovation among Spanish Manufacturing SMEs. Journal of Small Business Management, 47(4), 465-488.

Mancusi, M.L., \& Vezzulli, A. (2014). R\&D and Credit rationing in SMEs. Economic Inquiry, 52(3), $1153-1172$.

Mirow, C., Hoelzle, K., \& Gemuenden, H. (2008). The ambidextrous organization in practice: barriers to innovation within research and development. Academy of Management Proceedings, 1-6.

Mirow, C., Hölzle, K., \& Gemünden, H. (2007). Systematisierung, Erklärungsbeitrage und Effekte von Innovations barrieren . Journal für Betriebswirtschaft , 57(2), 101-134.

Mowery, D.C., Oxley, J.E., \& Silverman, B.S. (1996). Strategic alliances and interfirm knowledge transfer. Strategic Management Journal, 17(S2), 77-91.

Mowery, D., \& Oxley, J. (1995). Inward technology transfer and competitiveness: The role of national innovation systems. Cambridge Journal of Economics, 1, 67-93.

Neely, A., \& Hii, J. (1998). Innovation and business performance: a literature review. The Judge Institute of Management Studies, University of Cambridge, 1-57.

O'Brian, R. (2007). A caution regarding rules of thumb for variance inflation factors. Quality and Quantity, 41(5), 673-690.

OECD (2005a). The measurement of scientific and technological activities. En Proposed guidelines for collecting and interpreting technological data. Paris: Organisation for Economic Co-operation and Development.

OECD (2005b). Manual de Oslo: Guía para la recogida e interpretación de datos sobre innovación.

Oslo, Noruega: Organisation for Economic Co-operation and Development.

Oh, J.-M. (2017). Absorptive capacity, technology spillovers, and the cross-section of stock returns. Journal of Banking \& Finance, 85, 146-164.

Oltra, M.J., \& Flor, M. (2003). The Impact of Technological Opportunities and Innovative Capabilities on Firms' Output Innovation. Creativity and Innovation Management , 12(3), 137-144.

Palacios-Marqués, D., Soto-Acosta, P., \& Merigó, J.M. (2015). Online social networks as an enabler of innovation in organizations. Management Decision, 53(9), 1906-1920.

Paula, F., \& Silva, J. (2018). The impact of alliances and internal R\&D on the firm's innovation and financial performance . Brazilian Business Review, 15, 533-550.

Pellegrino, G., \& Savona, M. (2017). No money, no honey? Financial versus knowledge and demand constraints on innovation. Research Policy, 46(2), 510-521.

Pérez, E.O., \& Toro, I. (2018). Study of the realized absorptive capacity in the organizations of Colombia and Brazil. Journal of Industrial Engineering and Management, 11(4), 617-631.

Pisano, G. (2006). Can science be a business? Lessons from Biotech. Harvard Business Review, 84(10), 114-125.

René, B., Carree, M., Diederenc, B., Lokshin, B., \& Veugelers, R. (2004). Heterogeneity in R\&D cooperation strategies. International Journal of Industrial Organization, 22(8-9), 1237-1263. 
Richardson, G. (1972). The Organisation of Industry. The Economic Journal, 82(327), 883-896.

Sánchez, I., Juliao, J., \& Zuluaga, J. (2013). La relación entre las redes externas de trabajo y el desempeño innovador de las pymes colombianas: un análisis del rol moderador del ambiente industrial. Estudios Gerenciales, 29, 339-349.

Scuotto, V., Del Giudice, M., \& Carayannis, E. (2017). The effect of social networking sites and absorptive capacity on SMES' innovation performance. Journal of Technology Transfer, 42, 409-424.

Sekaran, U., \& Bougie, R. (2009). Research Methods for Business (Quinta ed.). Cornwall, United Kingdom: John Wiley \& Sons Ltd.

SENPLADES (2012). Transformación de la matriz productiva. Quito: Ediecuatorial.

Sok, P., \& O'Cass, A. (2011). Achieving superior innovation-based performance outcomes in SMEs through innovation resource-capability complementarity. Industrial Marketing Management, $40,1285-1293$.

Stock, G.N., Greis, N.P., \& Fischer, W.A. (2001). Absorptive capacity and new product development. The Journal of High Technology Management Research, 12(1), 77-91.

Tsai, W. (2001). Knowledge Transfer in Intraorganizational Networks: Effects of Network Position and Absorptive Capacity on Business Unit Innovation and Performance. Academy of Management Journal, 44(5), 996-1004.

Tsai, W. (2008). External technology acquisition and firm performance: A longitudinal study. Journal of Business Venturing, 23(1), 91-112.

Utterback, J.M., \& Abernathy, W.J. (1975). A dynamic model of process and product innovation . Omega, 3(6), 639-656.

Verhees, F., \& Meulenberg, M. (2004). Market orientation, innovativeness, product innovation, and performance in small firms. Journal of Small Business Management, 42(2), 134-154.

Wagner, T. (2013). Crear Innovadores. Bogotá, Colombia: Simon \& Schuster, Inc.

Wang, K., Hermens, A., Huang, K.-P., \& Chelliah, J. (2015). Entrepreneurial orientation and organizational learning on SME's innovation. International Journal of Organizational Innovation, 7(4), 65-75.

Weerawardena, J., Mort, G., Liesch, P., \& Knight, G. (2007). Conceptualizing accelerated internationalization in the born global firm: A dynamic capabilities perspective . Journal of World Business, 42(3), 294-306.

Woolthuis, R. K., Lankhuizen, M., \& Gilsing, V. (2005). A system failure framework for innovation policy design. Technovation, 25, 609-619.

Yam, R.C., Lo, W., Tang, E.P., \& Law, A.K. (2011). Analysis of sources of innovation, technological innovation capabilities, and performance: An empirical study of Hong Kong manufacturing industries. Research Policy, 40(3), 391-402.

Zahra, S., \& George, G. (2002). Absorptive capacity: A review, reconceptualization, and extension. Academy of Management Review, 27(2), 185-203.

Zhai, Y.-M., Sun, W.-Q., Tsai, S.-B., Wang, Z., Zhao, Y., \& Chen, Q. (2018). An Empirical Study on Entrepreneurial Orientation, Absorptive Capacity, and SMEs' Innovation Performance: A Sustainable Perspective. Sustainability, 10(2), 314. 